\section{WHAT DO WE MEAN BY TALKING ABOUT "VALUE(S)"? A REPLY TO SAARNI ET AL.}

doi:10.1017/5026646231200013X

In their article "Different methods for ethical analysis in health technology assessment: An empirical study" published in International Journal of Technology Assessment in Health Care, Volume 27 Number 4, Samuli I. Saarni, Annette BraunackMayer, Bjørn Hofmann, and Gert Jan van der Wilt present an empirical study about different methods for ethical analysis in health technology assessment (HTA). They claim that ethical analysis is an important issue which can highlight values inherent in a technology and value-decisions underlying a HTA process (4). Although their study is rich in detail; gives an interesting overview of casuistry, principlism, and axiological ethic frameworks; and make ethical evaluations within HTA more transparent, we suppose, one important point needs further serious discussion: the clarification of what is meant by "value."

The term value has different meanings depending on the discipline and point of view (5). There are, for example, moral values like justice, freedom or welfare, as well as non-moral values, for example, economic values (efficacy, efficiency, profit), scientific values (correctness, accuracy), or values in arts (e.g., harmony, aesthetic, balance). That means, everyone has a different perspective on the term "value." Physicians have another idea of value than health economists or patients have. Patients might be interested in leading a good life (healthy or not), physicians may have the goal to optimize health, and health economists compare costs and utility for society. Michael E. Porter defines patient value as the "health outcomes achieved by dollar spent" and states "that value should always be defined around the customer" (3). So, the question arises: Is value something subjective, which each and every person should define on his or her own? Or do objective values exist, which might be the basis for decision making? In (moral) philosophy, these questions are highly discussed, and many different value concepts have been evolved, one may distinguish between intrinsic and extrinsic forms of value, or discuss the priority of different values in different settings.

In assessing healthcare technologies, value is often defined as a kind of relation between outcome and costs - a kind of "trade-off." What is meant by costs is relatively clear (e.g., tangible or intangible costs). But when it comes to outcomes in health care, we have another important issue. Should we measure economic values like efficacy, effectiveness, efficiency, benefit or utility? The result you get depends heavily on the method you use to measure the outcomes - and its underlying value concept. As a consequence, this leads to another important issue: Are different values (e.g., economic, moral, or scientific values) even comparable? How should we handle conflicts between such value systems? Justice, health, a long life, autonomy, nonmaleficence, beneficence, etc.- these moral ideals or virtues are all values which may enable us to lead "a good life" (1). Philosophically, the definition of what is meant by "a good life" or "well-being" is a very challenging project (2). We live in pluralistic societies, where it is nearly impossible to define a universal basis of values for all. Which values are accepted as values depends on the socio-economic as well as the cultural background. This difficulty needs to be taken into consideration in technology assessment in health care, too.

So, first, it should be made clear what is exactly meant by value and, second, which method is taken to measure the "valued outcome(s)." Such a debate about value and its impact on technology assessment in health care is often missing or left aside. The different disciplines at work-for example, medicine, health economy, philosophy, (empirical) technology assessment - should once again think about what they mean when they refer to their different concepts of "value." Without such recurring, basic clarification, technology assessment in health care might lose important roots. 
Kathrin Dengler, Dipl-WiWi

email: kathrin.dengler@uni-ulm.de

Uta Bittner, MA

Institute of the History, Philosophy and Ethics of Medicine

Ulm University

Ulm, Germany

\section{CONFLICTS OF INTEREST}

Both authors report they have no potential conflicts of interest.

\section{REFERENCES}

1. Foot F. Virtues and vices. Oxford: Oxford University Press; 2002.

2. Griffin J. Well-being. Its meaning, measurement and moral importance. Oxford: Clarendon Press; 2002.

3. Porter ME. What is value in health care? N Engl J Med. 2010;363:24772481.

4. Saarni SI, Braunack-Mayer A, Hofmann B, van der Wilt GJ. Different methods for ethical analysis in health technology assessment: An empirical study. Int J Technol Assess Health Care. 2011;27:305312.

5. Scanlon TM. What we owe to each other. Cambridge, MA: Harvard University Press; 2000.

\section{TO EVALUATE VERSUS TO KNOW THE VALUE OF EVERYTHING}

doi:10.1017/50266462312000165

Kathrin Dengler and Uta Bittner demand a full-fledged philosophy of values in our empirical study of various methods for ethical analysis in health technology assessment (HTA). This may be like putting the classification of disease on hold until the concept of disease is clarified, or postponing the development of health care until the term "health" is clarified. As Dengler and Bittner rightly point out, the term value has many meanings, and as they properly recognize: "[P]hilosophically, the definition of what is meant by 'a good life' or 'well-being' is a very challenging project." Hence, it may be a bit over the top to crave that we solve eternal issues in an empirical article on methodology.

Furthermore, as we underscore in our article (4), the assessment and decision making context is important. "[T]he value-ladenness of a technology depends on the cultural context where it is applied." (5). Hence, values may be quite different in various settings, as may the meaning of value as such. As Dengler and Bittner rightly point out, there are many types of values (scientific, moral, aesthetic, economic) and values may be subjective and objective. We would like to add that values can be intrinsic and extrinsic (instrumental, inherent, contributory, relational, indicative), and they can be intersubjective. We do not demand universal definitions of "the good life" and "well-being" to address value issues in HTA, as Dengler and
Bittner do. This is because our main point is to highlight valuejudgments and value-issues in the assessment, implementation, and use of health technology in context. We do not want to impose our conceptions of values on patients, users, and decision makers. Instead, we want them to be aware of and reflect on value issues in the context where they themselves define value. In particular, we do not have the same strong preference for economic values as Dengler and Bittner. That being said, we do have some categories of values which we think are relevant to the assessment of health technology in most cases, such as general moral values, stakeholder interests, technological valueladenness, methodological values in HTA, and values related to HTA (and EBM) and a more fine grained explication of such values is also provided $(1 ; 3)$.

Although their call for of a philosophy of value analysis in our empirical article on ethics method in HTA may be demanding too much, the question itself is of course highly relevant and interesting. It has been dealt with in the philosophy literature repeatedly. However, as Dengler and Bittner presumably know, there is no consensus on the matter. Waiting on consensus before elaborating ethics methodology may be misguided. As we use terms such as health and disease without clear definitions (2), we may have to use terms such as value and good life with similar lack of definition. Moreover, we do not think it is wise to enforce stringent definitions of value on the contextual assessment. This may distract and hamper the reflection on values in the context where they are at play and, at the same time, defined.

Hence, we agree with Dengler and Bittner that the challenges with defining values need to be taken into consideration in technology assessment in health care, but we do not think it is necessary to do it top down. Their solutions and definitions are of course most welcome.

Bjørn Hofmann, $\mathrm{PhD}$

email:b.m.hofmann@medisin.uio.no

University College of Gjøvik and Norwegian Knowledge Center for the Health Services

Oslo, Norway

Samuli I. Saarni, MD, PhD

National Institute for Health and Welfare

Department of Mental Health and Substance Abuse Services

Helsinki, Finland

Department of Psychiatry

Helsinki University Central Hospital

Helsinki, Finland

Annette Braunack-Mayer, $\mathrm{PhD}$

Adelaide Health Technology Assessment School of Population

Health and Clinical Practice

University of Adelaide

Adelaide, Australia 